\author{
Julie Gard DVM, PhD.* \\ Department of Clinical Sciences, Auburn University \\ College of Veterinary Medicine, Auburn, Alabama, \\ USA \\ Dates: Received: 02 May, 2016; Accepted: 23 May \\ 2016; Published: 25 May, 2016 \\ *Corresponding author: Julie Gard DVM, PhD. \\ Department of Clinical Sciences, Auburn University \\ College of Veterinary Medicine, Auburn, Alabama, \\ USA, 36849, 334-844-4490,Tel: 334-321-7865; Fax: \\ 334-844-4368; E-mail: waldrja@auburn.edu \\ www.peertechz.com \\ Keywords: Campylobacter; Vibiosis; Veneral \\ disease; Cattle
}

Mini Review

\section{Bovine Genital Campylobacteriosis - A Review}

\section{Review}

Bovine genital campylobacteriosis (BGC) also termed bovine venereal campylobacteriosis (BVC) or Vibriosis is considered one of the two true venereal diseases of cattle [1-4]. Trichomoniasis caused by Tritichomonas fetus is considered the other true venereal disease of cattle $[2,3]$. The causal agent of the sexually transmissible disease, BGC, is Campylobacter fetus [1-4]. However, after further examination of Campylobacter fetus by microbiologist a division was made into two closely related subspecies: C. fetus subsp. venerealis and C. fetus subsp. fetus [4]. An additional subspecies of venerealis was identified as biotype intermedius (C. fetus venerealis intermedius) $[5,6]$. This biotype has also been shown to causes fertility issues in cattle $[5,6]$. The main causative agent of BCG is considered to be C. fetus subsp. venerealis (CFv) and infection is often manifested as infertility, embryonic and fetal death, metritis, salpingitis, pyometra, and abortion [2,3,6-8 ]. Abortions can occur at any time but are most commonly detected at 4 to 6 months of gestation [8]. Significant economic losses occur with infected herds especially in endemic areas $[2,6,8]$. Campylobacteriosis is a disease of socio-economic and public health significance and is a list $\mathrm{B}$ notifiable disease of the World Organization of Animal Health (OIE) [6]. Campylobacteriosis exists worldwide including Latin-America, Africa, Asia, North America, Europe, and NewZealand [6-10]. The prevelance of $C F v$ in some countries is still questionable due to lack of reporting and reliable testing $[9,10]$. Bovine infections with C. fetus subsp. fetus (CFf) do occur but are considered to be much more sporadic in occurrence in comparison to sheep. Oral transmission of CFf is occurs resulting in colonization the intestines of cattle and sheep leading to enteritis and abortion mostly in sheep and sporadically in cattle $[6,8]$.

C. fetus venerealis is a comma shaped microaerophilic, gramnegative rod. It often times has an "S" shape and has been described as having "spiral" or "seagull" shape [6]. The bacteria are well adapted to surviving in the low oxygen environment of the epithelial folds found in the prepuce of older bulls [11]. Isolation and identification of the agent, C. fetus venerealis, is the prescribed test for international trade according to the OIE [6]. The OIE details appropriate reporting, and testing for C. fetus. In order for Campyloacter to be detected on culture, the sampler needs to be cultured at $37^{\circ} \mathrm{C}$ for at least three days in a micro-aerobic atmosphere [6]. Most culture media contain the antifungal, cycloheximide, but due to its potential toxicity it is often replaced by amphotericin B [6]. The recommended selective medium for isolation of C. fetus is Skirrow's [6]. Skirrow's medium is a bloodbased medium with 5-7\% (lysed) defibrinated blood and contains the selective agents: polymyxin B sulphate $(2.5 \mathrm{IU} / \mathrm{ml})$, trimethoprim $(5 \mu \mathrm{g} / \mathrm{ml})$, vancomycin $(10 \mu \mathrm{g} / \mathrm{ml})$, and cycloheximide $(50 \mu \mathrm{g} / \mathrm{ml})$ or amphotericin B [6]. Additional discrimination between species and subspecies requires additional biochemical or molecular methods. The vaginal mucus from a cow that recently aborted can be tested via a vaginal mucus agglutination test or an ELISA. The ELISA can detect antibodies to IgA. Immunofluorescence may also be used to identify the organism from genital discharges of bulls, cows and heifers, but it will not differentiate between different subspecies $[6,8]$. Utilization of PCR for diagnosis has provided a way to easily differentiate between subspecies. However, not all PCRs are alike and so there have been mixed results [9-12]. The development of a new real-time quantitative polymerase chain reaction ( $\mathrm{qPCR}$ ) test has shown to be very reliable and was used to diagnose $C F v$ infection in a Canadian cattle herd [11]. This qPCR was verified with repeated culture, phenotypic characterization of the organism and DNA sequencing . Obviously, a reliable practical field test for $C F v$ would prove to be very useful but currently is not available.

Transmission of the organisms occurs most often during natural mating. However, transmission can also occur during artificial insemination with contaminated semen [1,6]. During natural exposure bulls are infected by carrier cows and or carrier bulls infect cows during coitus. During coitus contaminated semen gains entrance to the vagina and can live in the cervical mucus from three weeks to three months. During artificial insemination, the contaminated semen is placed within the uterus and / or very close to the internal cervical os and readily establishes uterine infection. The organism can grow and thrive on the penis and prepuce of the bull with the correct microenvironment. Specifically, $C F v$ is confined to the epithelial surface of the gland penis, prepuce, and the urethra [1]. It has been reported to live in the crypts of the prepuce [1-3,6]. However, Strickland et al., 2010 [13], reported following numerous preputial biopsies from a number of bulls, that there was insufficient 
gross or histological evidence to term these areas "crypts". Hence, it was suggested that the term "mucosal folds" be a utilized to more accurately describe these areas. Bulls do have numerous folds of the mucosa which seem to assist in the creation of the correct micro-environment on the prepuce, penis and urethra that allows for growth and sustainability of CFv. Chronic infections can be established within the prepuce and without causing observable signs which is seen most often in older bulls $[1-3,6,8]$. The chronic carriers propagate the disease within the herd. Some bulls, especially younger bulls, the infection may be transient and they may clear the infection but can become re-infected at a later date.

Susceptible females that are bred by $C F v$ carrier bulls can have customary conception rates [2,8]. However later in gestation, vaginitis, cervicitis, and then endometritis and salpingitis develops resulting in embryonic and fetal death at approximately seven to ten weeks of development $[2,8]$. Infected cows or heifers will develop a mucosal immune response approximately three to five months after the onset of infection. Most often this mucosal immune response will result in clearance of $C F v$ from the uterus and oviducts [8]. The reestablishment of normal fertility along with temporary immunity can follow along with resistance to subsequent infection. However, the immunity is somewhat short lived and lasts for approximately two years $[2,6]$. Persistent infections of $C F v$ can occur when convalescing females re-acquire a vaginal infection .

Diagnosis is made through collection of cervical mucus from infected cows and heifers often collected at three weeks to three months post infection. Culture results, vaginal mucus agglutination tests, fluorescent antibody tests can all be questionable so confirmatory PCR is being looked to as a more reliable diagnostic of herd infection $[8,11,12]$. Also, if cervical mucus sampling is not completed in estrus but in metestrus false positives can occur at a higher rate $[8,12]$. Diagnosis in the bull is performed most often via a preputial scraping or preputial wash performed twice one week apart or testing of contaminated semen via culture, fluorescent antibody and PCR $[6,12]$. It is imperative that $C F v$ should be inoculated into Clark's media or Skirrow's media soon after collection of the sample to allow for maximum survival of the organism $[6,8]$. The organism can survive for only a short period of time, approximately six to eight hours following collection. However, if the sample is placed in Clark's media for transportation and / or culture it will allow for a longer period of survival up to 48 hours.

Whole herd treatment of $C F v$ is considered to be impractical due to the lack of overt clinical signs at the initiation of the disease process [8]. However if elected, bulls may be treated by administering one to two treatments of streptomycin given subcutaneously at a dose of $20 \mathrm{mg} / \mathrm{kg}$ along with administration of a topical suspension containing five grams of streptomycin to the prepuce and penis for three consecutive days but resistant strains have been reported [8]. Hence, prevention of venereal diseases in the herd is the most practical approach to dealing with $C F v$ as well as $T$. foetus $[1,2]$. Control measures include utilization of artificial insemination with non-contaminated semen, testing and culling of infected animals, and vaccination of all bulls, cows and heifers $[2,6,8,14,15]$. Utilization of artificial insemination exclusively for two breeding seasons is suggested in infected herds due to the fact that $C F v$ has been isolated from cows for greater than six months after calving [8]. However, total use of artificial insemination is impractical in range cattle. Hence, most producers utilize management, culling problem cattle and use of virgin bulls, and vaccination as their primary means of prevention and control [8].

The recommendations for vaccination by the OIE includes vaccination of all infected herds, all breeding animals, bulls, cows and heifers should be vaccinated twice prior to the breeding season except in countries that have been determined to be free of C. fetus. Vaccination is prohibited in C.fetus free countries [6]. In order to stimulate effective immunity, it is imperative to induce IgA in the vagina and IgG in the uterus $[2,6,14]$. Hence, cows and heifers are often administered an additional vaccine in the middle of the breeding season to enhance their immunity. Vaccination reduces the length of the infection but carrier-cows have been known to harbor the infection from one season to the next and have the potential to infect susceptible bulls $[6,14,15]$. Also, it has been suggested that bulls should be given double the dose of vaccine at each of two vaccinations annually to assist in terminating established infections $[6,14]$. However, in non-infected herds the bulls should at least be vaccinated initially with two doses given 3-4 weeks apart with the last dose approximately 30 days prior to the start of the breeding season $[6,8]$.

There has been controversy in use of vaccines and the efficacy of them especially in bulls. Vaccination against C. fetus subsp. veneralis has been reported to be protective and even curative for the bull and cow $[4,14,16]$. Although, other studies have conveyed that prevention of infection utilizing commercial vaccinations has had questionable efficacy [8,14]. A recent study by Cobo et al., 2004 [14], evaluated an experimental vaccine containing Campylobacter fetus and Tritrichomonas foetus antigens compared to commercial vaccine utilizing subcutaneous and intravaginal administration routes. It was found that heifers vaccinated with the experimental vaccine had an increased clearance rate of both pathogens, higher pregnancy rates and higher systemic immune responses during and after breeding. Hence, it was determined that the experimental vaccine was superior to the commercial vaccine with both bovine venereal campylobacteriosis and trichomonosis were tested. So, vaccination especially for $C$. fetus has been found to beneficial $[2,6,8,14]$. It is important that vaccines contain specific antigens, the thermolabile ' $\mathrm{H}$ ' flagellar antigens and the thermostable ' $\mathrm{O}$ ' somatic antigens, the capsular ' $\mathrm{K}$ ' antigens and four to five heat-labile glycoprotein immunogens which are shared by many C. fetus subsp. venerealis and C. fetus subsp. fetus strains $[6,14]$.

For those countries free of $C$. fetus such as the member countries of the European Union or wanting to decrease the incidence or to become C. fetus free the OIE has outlined management protocols that include importation of breeding females, bulls and semen [17]. All animals need to be accompanied by an international veterinary certificate stating that all animals were virgins and kept in a $C$. fetus negative herd where there have been no cases of $C$. fetus. If the animals are bred then the vaginal mucus or semen, or preputial smegma need to be tested negative for C. fetus prior to importation. 
Additionally, imported semen must come from A-I Centers that are free of $C$. fetus. Artificial insemination is a tool for control and management of $C f v$. Countries that are free of C. fetus do not usually employ vaccination in management protocols due to the possibility of masking disease outbreaks. It is important in maintaining free status to quickly identify disease in the population and prevent the disease from spreading further. Screening tests which have a high sensitivity are implemented as the first line of testing in control and eradication programs. Tests which have a high specificity are necessary in the second line of testing to determine which animals are false positive in those testing positive on screening tests. The positive predictive value depends on the prevalence of the disease in the population so the probability of disease among subjects with positive tests will be higher.

\section{References}

1. Eaglesome M, Garcia M (1992) Microbial agents associated with bovine genital tract infections and semen. Part I. Brucella abortus, Leptospira, Campylobacter fetus and Tritrichomonas foetus. Vet Bull 62: 743-775.

2. Bondurant RH (2005) Venereal diseases of cattle: Natural history, diagnosis, and the role of vaccines in their control. Vet Clin North Am Food Anim Pract 21: 383-408.

3. Skirrow S, BonDurant R (1998) Bovine trichomoniasis. Vet Bull 58: 591-603.

4. Clark B, Dufty J, Monsbourgh M (1968) Experimental Vibrio fetus (venerealis) infection in heifers. The immunising properties of killed organisms injected subcutaneously. Aust Vet J 44: 110-114.

5. MacLaren AP, Agumbah GJ (1988) Infertility in cattle in south-west Scotland caused by an "intermediate" strain of Campylobacter fetus subspecies fetus (formerly Campylobacter fetus intestinalis). Br Vet J 144: 29-44.

6. OIE; http://www.oie.int/fileadmin/Home/eng/Health_standards/tahm/2.04.05 BGC.pdf Chapter 2.4.5

7. Cipolla A, Paolicchi F, Poso M, Morsella C, Casaro A, et al. (1998) Lectin- bindings sites in uterus an oviduct of normal and Campylobacter fetus subspecies venerealis-infected heifers. Eur J Histochem 42: 63-70.

8. Truyers I, Luke T, Wilson D, Sargison N (2014) Diagnosis and management of venereal campylobacteriosis in beef cattle. BMC Veterinary Research 10: 280

9. Sanhueza JM, Heuer C, Jackson R, Hughes P, Anderson P, et al. (2014) Pregnancy rates of beef cattle are not affected by Campylobacter fetus subsp. venerealis real-time PCR-positive breeding sires in New Zealand. NZ Vet J 62: 237-243.

10. Hosseinzadeh S, Kafi M, Pour-Teimouri M (2013) PCR detection of Campylobacter fetus subspecies venerealis in smegma samples collected from dairy cattle in Fars, Iran. Vet Res Forum 4: 227-231.

11. Waldner C, Hendrick S, Chaban B, Garcia Guerra A, Griffin G, et al (2013) Application of a new diagnostic approach to a bovine genital campylobacteriosis outbreak in a Saskatchewan beef herd. Can Vet J 54: 373-376.

12. Chaban B, Chu S, Hendrick S, Waldner C, Hill (2012) Evaluation of a Campylobacter fetus subspecies venerealis real-time quantitative polymerase chain reaction for direct analysis of bovine preputial samples. Can J Vet Res 76: 166-173.

13. Strickland L (2010) Surface Architectural Anatomy of The Penile and Preputial Epithelium of Bulls. Thesis 2010.

14. Coboa ER, Morsellac C, Canoc D, Cipollac A, Camperoc CM (2004) Immunization in heifers with dual vaccines containing Tritrichomonas foetus andCampylobacter fetus antigens using systemic and mucosal routes. Theriogenology 62: 1367-1382.

15. Sanderson M, Gnad D (2002) Biosecurity for reproductive diseases. Vet Clin Food Anim 18: 79-98.

16. Yaeger MJ, Holler LD (2007) Bacterial causes of bovine infertility and abortion. In: Younquist R.S., editor. Current Therapy in Large Animal Theriogenology. 2nd ed. Philadelphia; W.B. Saunders Company 391.

17. OIE (2015) http://www.oie.int/fileadmin/Home/eng/Health_standards/ tahc/2010/chapitre_bovine_genital_campylobacteriosis.pdf. Chapter 11.3 of the Terrestrial Animal Health Code. 